\title{
Excitation structure in a Micro Hollow Cathode Discharge in the normal regime at medium argon pressure
}

\author{
C.Lazzaroni ${ }^{1}$, P. Chabert ${ }^{1}$, A. Rousseau ${ }^{1}$ and N. Sadeghi ${ }^{2}$ \\ ${ }^{1}$ Laboratoire de Physique des Plasmas, Ecole Polytechnique, CNRS, 91128 Palaiseau, France \\ ${ }^{2}$ Laboratoire de Spectrométrie Physique, Univ. Joseph Fourier \& CNRS, Grenoble, France \\ E-mail: claudia.lazzaroni@lpp.polytechnique.fr
}

\begin{abstract}
A microplasma is generated in the microhole ( $400 \mu \mathrm{m}$ diameter) of a molybdenum-aluminamolybdenum sandwich (MHCD type) at medium pressure (30-200 Torr) in pure argon. Imaging and emission spectroscopy have been used to study the light emission mechanisms in the microdischarge. We find that emission intensities of both argon atom and argon ion lines present sharp peaks located near the cathode, and that the position of these peaks moves toward the cathode with increasing gas pressure. An ionizing-sheath model has been developed to calculate the evolution of the sheath thickness as a function of the gas pressure. This model includes the ratio between the ion flux coming from the bulk of the plasma and the ion flux created in the sheath and also accounts for the gas heating in the microhole. The variation of the calculated ionizing sheath thickness as a function of pressure agrees well with the variation of the measured peak positions. The case of a sheath free of ionization has also been considered. However, the comparison between experiments and the various theories shows that a significant fraction of the ionization occurs in the sheath.
\end{abstract}

\section{Introduction}

The study of microplasma began to develop in the middle of the 90's in the USA [1], before undergoing an important expansion thanks to the experimental and theoretical works that have been performed in the past few years [2, 3, 4, 5 and 6]. The interest of microplasmas is that they can be generated at medium and high pressure (atmospheric pressure) with a very low applied voltage or injected power compared with others non-thermal plasmas such as dielectric barrier discharges or corona. They have applications in various fields such as surface treatment [7], sterilization [8], light sources [9] or microjets [10]. A particular microplasma configuration is the hollow cathode type geometry where a hole is drilled through an electrode-dielectric-electrode sandwich structure. This device is particularly stable. At very low current (typically $<0.1 \mathrm{~mA}$ in our device), the plasma is located inside the hole and the voltage increases with the current: this is the abnormal regime. However, despite a DC driving source, a self-pulsed regime appears for intermediate discharge currents (typically $\leq 0.5 \mathrm{~mA}$ time averaged current for our device) with frequency of several tens of kilohertz [11]. This regime corresponds to a periodic plasma expansion on the cathode surface [12]. At higher currents, the plasma expands on the cathode backside surface and the voltage becomes relatively independent of the discharge current: this is the normal regime.

In this paper, we study the excitation mechanisms of the MHCD structure during the normal regime thanks to the observed radial profiles of argon atom and argon ion emission line intensities. A theoretical calculation of the sheath thickness is proposed to help a better understanding of the experimental results.

\section{Experimental set-up}

The microplasma device shown in figure 1 is similar to the one developed by Schoenbach [1]. It is a metal-dielectric-metal sandwich (microhollow cathode type). The dielectric, constituted of a $250 \mu \mathrm{m}$ alumina foil, separates the two molybdenum electrodes which are both $L=150 \mu \mathrm{m}$ thick foils. A $\mathrm{R}=200 \mu \mathrm{m}$ radius hole is drilled through the three foils with a laser beam, which are subsequently glued to form the sandwich structure. A ballast resistor, $R_{b}=440 \mathrm{k} \Omega$ is located on the cathode side and a 
measurement resistor, $\mathrm{R}_{\mathrm{m}}=100 \Omega$ is located on the anode side. The discharge voltage is measured on the cathode side with a high voltage probe and the discharge current is deduced from the voltage drop across $R_{\mathrm{m}}$. These two signals are recorded on a 350MHz oscilloscope (Lecroy 6030A).

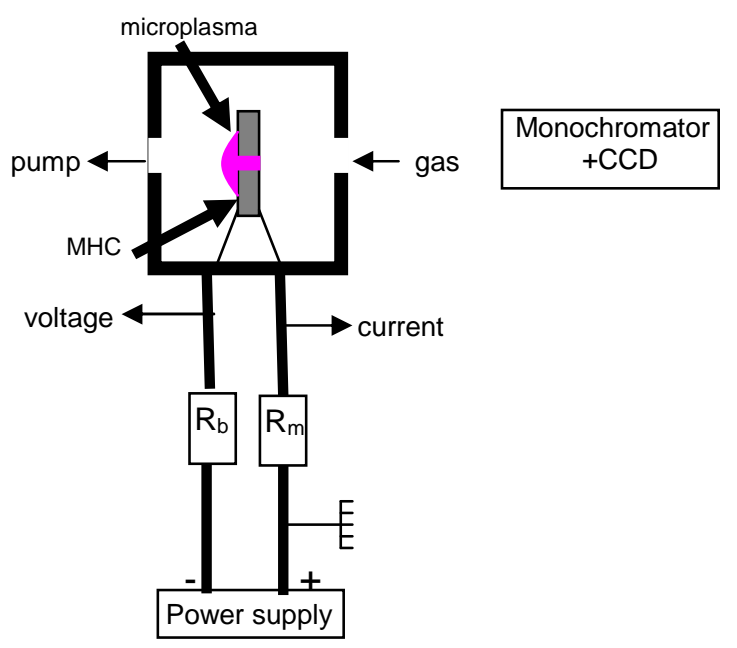

FIGURE 1. Experimental set-up.

Experiments reported in this paper are run in stable DC discharge conditions at constant argon flow of $100 \mathrm{sccm}$. Argon gas pressure ranges from 30 to 200 Torr and the discharge current is fixed at $1 \mathrm{~mA}$. Optical emission spectroscopy measurements are performed to investigate the plasma excitation structure of the micro-discharge and also to evaluate the gas temperature across the radius of the hole. The image of the MHCD, viewed from the anode side, is formed with an achromatic $12.5 \mathrm{~mm}$ diameter, $\mathrm{f}=30 \mathrm{~mm}$, lens (with an enlargement factor of about 8) on the entrance slit of a grating spectrograph. To record argon atomic and ionic lines in the $427 \mathrm{~nm}$ spectral region, a $2 \mathrm{~m}$ focal length spectrograph working at the third diffraction order of the $1200 \mathrm{~g} / \mathrm{mm}$ grating is used. To record the nitrogen first positive emission around $670 \mathrm{~nm}$, from which the gas temperature is deduced, a $1 \mathrm{~m}$ focal length spectrograph working at the first diffraction order of the $1200 \mathrm{~g} / \mathrm{mm}$ grating is used. In both cases, a CCD camera, with pixels size $16 \times 16 \mu \mathrm{m}^{2}$, placed on the exit plane of the spectrographs captures the horizontally dispersed images at different spectral positions. Therefore, with fully open entrance slit, images of the MHCD observed at different wavelengths are formed at different horizontal positions of the CCD. Each pixel of these images corresponds to an area of about $2.0 \mu \mathrm{m} \times 2.0 \mu \mathrm{m}$ inside the MHCD. Considering the spherical aberrations of the whole optical system, the spatial resolution along the diameter of the hole is estimated to be about $5 \mu \mathrm{m}$. The spectral dispersion of the $2 \mathrm{~m}$ spectrograph is $1.325 \mathrm{pm} / \mathrm{pixel}$ at $427.5 \mathrm{~nm}$ (around argon lines) and that of the 1 $\mathrm{m}$ spectrograph is $11.39 \mathrm{pm} /$ pixel at $670 \mathrm{~nm}\left(\mathrm{~N}_{2}\right.$ emission).

\section{Experimental results}

\subsection{Spatial distribution of $\mathrm{Ar}$ and $\mathrm{Ar}^{+}$lines emission intensities}

Figure 2 shows the CCD image of the $400 \mu \mathrm{m}$ diameter MHCD (100 Torr, $1 \mathrm{~mA}$ ) recorded with fully open entrance slit. The spectrograph is set to form images from both $\operatorname{Ar}\left(5 \mathrm{p}[3 / 2]_{1}-4 \mathrm{~s}[3 / 2]_{1}\right)$ line at $427.217 \mathrm{~nm}$ (left), and $\mathrm{Ar}^{+}\left(4 \mathrm{p}^{, 2} \mathrm{P}_{3 / 2}-4 \mathrm{~s}^{, 2} \mathrm{D}_{5 / 2}\right)$ line at $427.752 \mathrm{~nm}$ (right) on the CCD surface. As seen in this figure, the intensity of the $\mathrm{Ar}^{+}$emission line has an annular shape, with its maximum intensity near the cathode surface, whereas the intensity of the Ar emission line shows a pronounced maximum at the center of the MHCD hole. 
FIGURE 2. CCD imaging of $\mathrm{Ar} 427.217 \mathrm{~nm}$ (left) and $\mathrm{Ar}^{+} 427.752 \mathrm{~nm}$ (right) lines in the $400 \mu \mathrm{m}$ diameter hole, at 100 Torr argon pressure and 1mA discharge current.

With the entrance slit set at $100 \mu \mathrm{m}$ and the diameter of the MHCD image aligned on this slit, vertical line shape images at two spectral positions of $427.217 \mathrm{~nm}$ and $427.752 \mathrm{~nm}$ lines provide the radial profiles of the emission intensity of $\mathrm{Ar}$ and $\mathrm{Ar}^{+}$lines, respectively. Radial profiles recorded in the 400 $\mu \mathrm{m}$ hole at $1 \mathrm{~mA}$ discharge current and argon pressures of 30, 50, 100, 150 and 200 Torr are shown on figure 3 .
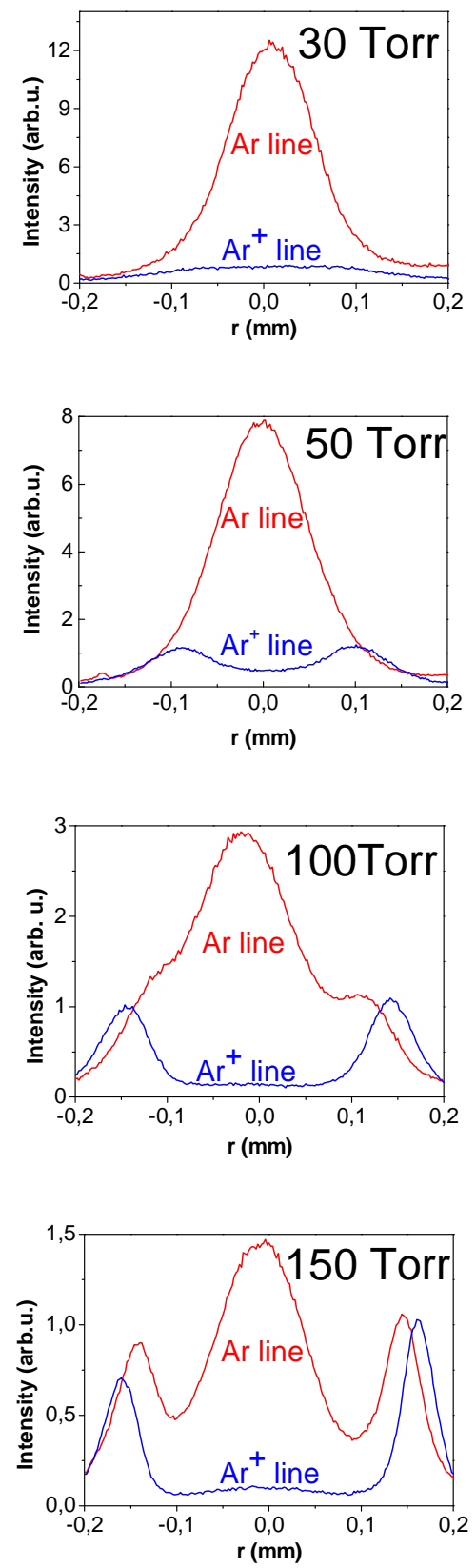


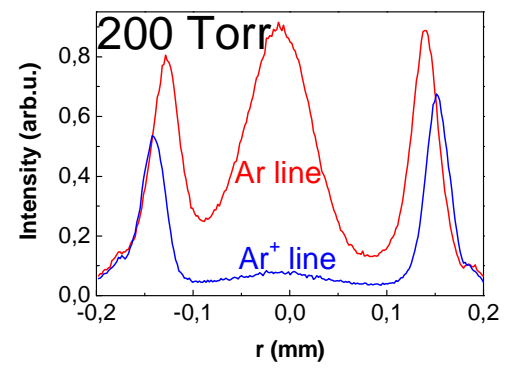

FIGURE 3. Radial profiles of the emission intensity of $\mathrm{Ar} 427,217 \mathrm{~nm}$ and $\mathrm{Ar}^{+}$427,752 $\mathrm{nm}$ lines in the $400 \mu \mathrm{m}$ diameter hole at $1 \mathrm{~mA}$ and 30, 50, 100, 150 and 200 Torr argon pressure.

The observed light from the micro-hole comes from the following mechanisms:

$$
\begin{cases}A r+e^{-} \rightarrow A r^{*}+e^{-} & \left(R_{1}\right) \\ A r+e^{-} \rightarrow A r^{+*}+2 e^{-} & \left(R_{2}\right) \\ A r+e^{-} \rightarrow A r^{+}+2 e^{-} & \left(R_{3}\right) \\ A r^{+}+2 A r \rightarrow A r_{2}^{+}+A r & \left(R_{4}\right) \\ A r^{+}+2 e^{-} \rightarrow A r^{*}+e^{-} & \left(R_{5}\right) \\ A r_{2}^{+}+e^{-} \rightarrow A r^{*}+A r & \left(R_{6}\right)\end{cases}
$$

The first two reactions are direct electron impact excitations from the ground state argon atoms to the observed excited states of $\mathrm{Ar}$ and $\mathrm{Ar}^{+}$, respectively. In the plasma bulk, $\mathrm{Ar}^{+}$density is at least $10^{5}$ times smaller than the Ar density. Due to the acceleration and flux conservation of ions, the density ratio will even be much larger in the sheath. The peak cross section for the excitation of the upper level of ion line is about $10^{-21} \mathrm{~m}^{-2}$ at $40 \mathrm{eV}$ [13]. The cross section for the excitation of this level from the ground state of ion can be at maximum 10 times larger than this value, with a threshold of about 20 $\mathrm{eV}$. Considering the high energy of beam electrons inside the sheath, the small differences in cross sections and excitation threshold cannot compensate more than five orders of magnitude in densities and consequently the excitation of $\mathrm{Ar}^{+}$line from the ground state of ion will be negligible. $\mathrm{R}_{3}$ is the ground state $\mathrm{Ar}^{+}$ion production from the ground state of atom and considering the high pressure domain of the MHCD, these $\mathrm{Ar}^{+}$ions are rapidly converted into $\mathrm{Ar}_{2}{ }^{+}$ions, with a rate coefficient for the reaction $\left(\mathrm{R}_{4}\right) \mathrm{k}_{4}=2.7 \times 10^{-43} \mathrm{~m}^{6} \cdot \mathrm{s}^{-1}$ [14]. The last two reactions are electron-ion recombinations involving $\mathrm{Ar}^{+}$and $\mathrm{Ar}_{2}{ }^{+}$ions. In our MHCD plasmas, with an electron density $\mathrm{n}_{\mathrm{e}} \simeq 10^{20} \mathrm{~m}^{-3}$, and electron temperature $\mathrm{T}_{\mathrm{e}} \cong 1 \mathrm{eV}$, the recombination coefficients of $\mathrm{Ar}^{+}$ions [15] $a_{5} \cong 5.6 \times 10^{-39} \mathrm{~T}_{\mathrm{e}}(\mathrm{eV})^{-9 / 2} \mathrm{n}_{\mathrm{e}} \cong 5 \mathrm{x}$ $10^{-19} \mathrm{~m}^{3} \cdot \mathrm{s}^{-1}$ is much smaller than that of $\mathrm{Ar}_{2}{ }^{+}$ions [16] $a_{6} \cong 8.5 \times 10^{-13}\left(\mathrm{~T}_{\mathrm{e}}(\mathrm{kT}) / 300\right)^{-2 / 3} \cong 7 \times 10^{-14} \mathrm{~m}^{3} \cdot \mathrm{s}^{-1}$. In conclusion, under our experimental conditions the dominant e-ion recombination mechanism that contributes to the production of excited Ar atoms is through $R_{4}$ and $R_{6}$ channels. The maximum of the emission intensity of atomic line at the MHCD centre observed in Fig. 3 for $p \geq 100$ Torr comes from excited $\mathrm{Ar}$ atoms produced by electron-ion recombination mechanism and as expected the maximum of the intensity is located at the central region of the MHCD, where the charged particle densities are maximum [17].

At 100, 150 and 200 Torr, the peaks observed for the two lines near walls of the MHCD may be related to the position of the sheath edge. Indeed, electrons emitted from the cathode are accelerated within the sheath, where the electric field is large, and release this energy away from the cathode. The exact location of the maximum of emission and ionization may only be calculated from kinetic theory. Since ionization in the sheath is required to sustain a DC discharge, we expect ionization and excitation in the sheath. However, the maximum of excitation may be located after the sheath edge, as we shall see in the next section. Since the threshold energy for the electronic excitation of the upper level of the $427.752 \mathrm{~nm} \mathrm{Ar}^{+}$line $\left(\mathrm{R}_{2}\right)$ is about $37 \mathrm{eV}$, i.e. much larger than that for the excitation of the $427,217 \mathrm{~nm}$ atomic line $\left(\mathrm{R}_{1}\right)$, we expect the maximum of $\mathrm{Ar}^{+}$line to come first. Indeed, we experimentally observe that the maximum of Ar line emission arises 10 to $20 \mu \mathrm{m}$ further away from 
the cathode compared to the ionic line. At lower pressures (50 Torr and below), the sheath size is no longer negligible compared to the hole radius and thus the maxima from both sides approach the centre and form a single maximum (hollow cathode effect). However, for $\mathrm{Ar}^{+}$line a maximum around $\mathrm{r}=100 \mu \mathrm{m}$ at 50 Torr and a shoulder around $\mathrm{r}=50 \mu \mathrm{m}$ at 30 Torr are also visible in figure 3 .

Also, we observe that when increasing the neutral gas pressure, the maxima near the cathode of both ionic and atomic lines move towards the cathode, which suggests that the sheath size shrinks. To verify this hypothesis and quantify the effect, we perform an analytical calculation of the sheath size. We shall see that the model requires the knowledge of the neutral gas temperature. This temperature has been measured and the results are presented in the next section.

\subsection{Measurement of the gas temperature}

The gas temperature is deduced from the population distribution in rotational level of $\mathrm{N}_{2}\left(\mathrm{~B}^{3} \Pi_{\mathrm{g}}, v=4\right.$ and 5) by recording the emission spectra of the first positive nitrogen system $N_{2}\left(B^{3} \Pi_{g}\right) \rightarrow N_{2}\left(A^{3} \Sigma_{u}\right)$. A small amount (less than 1\%) of nitrogen has been added to the feedstock gas and the recorded emission spectra of the 4-1 and 5-2 bands have been fitted with a simulated spectra (home made code using the spectroscopic constants of [18]) in order to obtain the rotational temperature. As an example, we show on figure 4, the recorded spectra from the emission at the MHCD axis for a pressure of 100 Torr and a current of $1 \mathrm{~mA}$. The best simulated spectra for a gas temperature of $470 \mathrm{~K}$, also shown on figure 4, do not include the 6-3 band whose rotational lines start down from the $665 \mathrm{~nm}$ region.

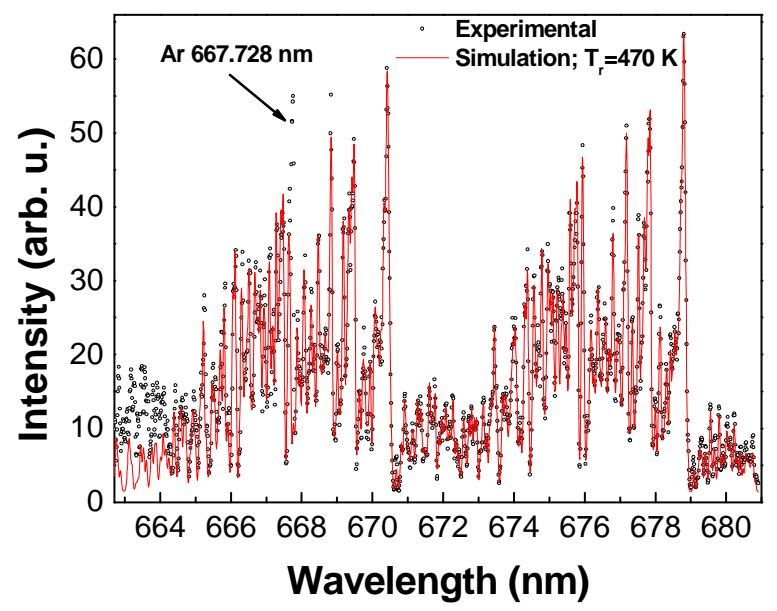

FIGURE 4. Experimental (black point) and simulated (red line) emission spectra in the $400 \mu \mathrm{m}$ diameter hole for a pressure of 100 Torr and a $1 \mathrm{~mA}$ discharge current.

The rotational temperature is supposed to be equal to the translational gas temperature because the rotational population of this excited state reaches thermal equilibrium before its radiative deexcitation. This is due to the long radiative life time of the $\mathrm{N}_{2}\left(\mathrm{~B}^{3} \Pi_{\mathrm{g}}\right)$ state (a few $\mu \mathrm{s}$ [19]) compared to the large collision frequency at the high argon gas pressure in our experiments.

The radial dependence of the gas temperature, obtained by fitting the observed spectra at different radial positions, is relatively constant along the MHCD diameter $( \pm 20 \mathrm{~K})$. This is probably because the gas heating is localized near the MHCD walls and that some of the heat transfer occurs along the axial direction. At 100 Torr and $1 \mathrm{~mA}$ current, the gas temperature is about $470 \mathrm{~K} \pm 10 \mathrm{~K}$ and it is only weakly dependent on gas pressure. However, as expected, the gas temperature increases with the discharge current. 


\section{Calculation of the sheath thickness}

\subsection{Case of an ionizing sheath}

We start our analysis by considering an ionizing sheath for which the analytical calculation of the sheath size is based on the well-known physics of the cathode fall in DC discharges (see for example [20] chapter 6). In accordance with our experimental observations, we will assume that electron-ion recombination is a negligible process inside the sheath and we do not account for it in the charged particles balance. Indeed, in the sheath, the velocity of both particles is very large compared to the thermal velocities and therefore the recombination rate is much smaller there. Besides, the electron density is too low inside the sheath to have a signification recombination process.

We consider a cylindrical geometry, with the parameters shown on figure 5:

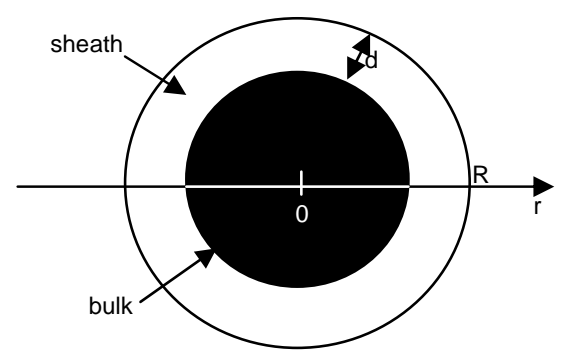

FIGURE 5. Schematic structure of the MHCD with a cylindrical cathode of radius R, separated from the plasma (in black) by an annular sheath of thickness $\mathrm{d}$.

Electrons emitted from the cathode at $\mathrm{r}=\mathrm{R}$ are accelerated by an electric field, ionizing the neutral gas background. The increase of the radial electron flux $\Gamma_{\mathrm{e}}$ from the cathode to the bulk is given by the following equation:

$$
\frac{1}{r} \frac{d\left(r \Gamma_{e}\right)}{d r}=-\alpha(r) \Gamma_{e}
$$

where we have assumed a one-dimentional cylindrical geometry, and where $\alpha(r)$ is the first Townsend coefficient and represents the inverse of an « ionization » mean free path.

This equation is integrated between R-d and R to obtain:

$$
\Gamma_{e}(R-d)=\Gamma_{e}(R)\left[\frac{R}{R-d} \exp \left(\int_{R-d}^{R} \alpha(r) d r\right)\right]
$$

Writing the continuity of total charge, the electron current $I_{e}(R-d)$ leaving the sheath edge at $\mathrm{r}=\mathrm{R}-\mathrm{d}$ minus the electron current $I_{e}(R)$ emitted from the cathode, is equal to the ion current $I_{i}(R)$ striking the cathode at $\mathrm{r}=\mathrm{R}$ minus the ion current $I_{i}(R-d)$ entering the sheath at $\mathrm{r}=\mathrm{R}$-d such that the fluxes obey the following equation:

$R \Gamma_{i}(R)-(R-d) \Gamma_{i}(R-d)=(R-d) \Gamma_{e}(R-d)-R \Gamma_{e}(R)$

Moreover, $\Gamma_{e}(R)=\gamma \Gamma_{i}(R)$, where $\gamma$ is the secondary emission coefficient of the cathode material. The ion current entering the sheath is considered to be a fraction (to be estimated) of the ion current at the cathode: $(R-d) \Gamma_{i}(R-d)=\xi R \Gamma_{i}(R)$. This fraction $\xi$ is supposed to be smaller than 0.5 in order to keep an ionizing sheath. Indeed, when $\xi=0$, the flux entering the sheath is negligible compared to the flux produced in the sheath (by ionization). This is the typical regime of the cathode fall in a DC discharge tube. On the other hand, when $\xi=1$, the flux produced in the sheath is negligible and this model breaks down. This case, typical of low-pressure wave-excited discharges where the sheath is usually free of ionization, is considered in the next section.

Expressing $\Gamma_{e}(R-d)$ as a function of $\Gamma_{e}(R)$ leads to the following integral form: 


$$
\int_{R-d}^{R} \alpha(r) d r=\ln \left(\frac{1-\xi}{\gamma}+1\right)
$$

At this point, we have a relation between $\alpha(r)$ and d, the sheath thickness that we wish to calculate. We then need an expression of $\alpha(r)$ in order to calculate the integral (4). The coefficient $\alpha$ is a complicated function of the pressure and of the accelerating field which is very difficult to calculate self-consistently. However, $\alpha$ might be expected to be expressed in the form [21]:

$\frac{\alpha}{p}=A \beta \exp \left(-\frac{B \beta p}{E}\right) \quad$ with $\beta=\frac{300}{T(K)}$

where $\mathrm{p}$ is the pressure measured experimentally and $\mathrm{E}$ the electric field. The factor $\beta$ accounts for higher gas temperature, and therefore lower neutral gas density, inside the micro-hole. The constants A and $\mathrm{B}$ are determined experimentally for each gas and found to be roughly constant over a restricted range of $\mathrm{E} / \mathrm{p}$ for any given gas [20]. For an argon gas, $\mathrm{A}=11.5 \mathrm{~cm}^{-1}$. Torr ${ }^{-1}$ and $\mathrm{B}=176 \mathrm{~V} \cdot \mathrm{cm}^{-1}$. Torr ${ }^{-1}$, for a range of $\mathrm{E} / \mathrm{p}$ of 100-600 V.cm ${ }^{-1}$.Torr ${ }^{-1}$ [20].

The next step is to determine the radial evolution of the electric field $\mathrm{E}$ within the sheath. Here we use the approximation of a high-voltage matrix sheath, that is uniform ion density and electron density essentially equal to zero. This approximation gives in Cartesian coordinates, a linear field variation with the distance from the cathode. This variation has been confirmed experimentally by the direct measurement of $E$ [22] or by observing the deflecting of a electron beam projected across the sheath, the deflection being proportional to the electric field [21]. Using the matrix sheath approximation and the Poisson's equation in cylindrical coordinates, the expression of the electric field $\mathrm{E}$ as a function of $\mathrm{r}$ is:

$$
E(r)=E_{0} \frac{R}{2 R d-d^{2}}\left(r-\frac{(R-d)^{2}}{r}\right)
$$

where $E_{0}$ is the value of the electric field at the cathode ( $\left.\mathrm{r}=\mathrm{R}\right)$. Due to the cylindrical geometry, the electric field does not vary linearly in space, unless in the case $d<<R$.

Using the relation between the electric field and the electric potential $\mathrm{V}, \vec{E}=-\vec{\nabla} V$, and integrating it between R-d to R, we find the relation between $E_{0}$ and the cathode sheath voltage $V_{c}$ :

$$
E_{0}=\frac{2 R d-d^{2}}{R} \frac{2}{d(2 R-d)+2(R-d)^{2} \ln \frac{R-d}{R}} V_{c}
$$

We finally substitute the expression of $\alpha$ as a function of $V_{c}$ in equation (4) to obtain:

$$
\ln \left(\frac{1-\xi}{\gamma}+1\right)=\int_{R-d}^{R} A \beta p \exp \left[-\frac{B \beta p}{2 V_{C}}\left(d(2 R-d)+2(R-d)^{2} \ln \frac{R-d}{R}\right) \frac{1}{r-\frac{(R-d)^{2}}{r}}\right] d r
$$

This integral equation is solved numerically to obtain the sheath thickness for each neutral gas pressure, with a secondary emission coefficient of $\gamma=0.07$ [4], a cathode sheath voltage $\mathrm{V}_{\mathrm{C}}$ of $250 \mathrm{~V}$ and a radius of the MHCD of $200 \mu \mathrm{m}$.

A plasma transport model is required to evaluate the parameter $\xi$, which represents the fraction of the flux produced in the plasma to the flux produced within the sheath. In this work, we keep $\xi$ as a parameter and evaluate its influence on the calculated sheath thickness d.

The theoretical values of the sheath thickness are compared with the experimental results on figure 6 for three values of $\xi$ between 0 and 0.5 : 


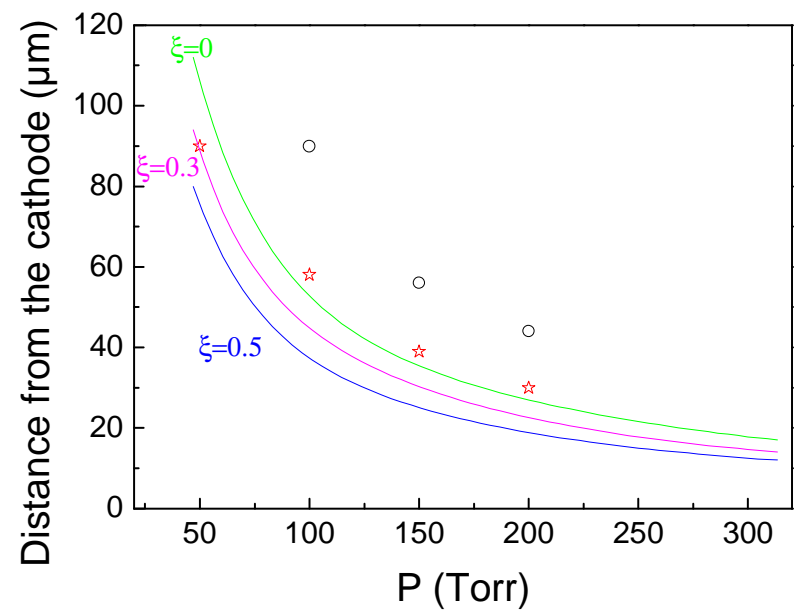

FIGURE 6. Pressure dependence of the distance from the cathode of the electron excitation maxima of atomic (black open circles) and ionic (red open stars) lines, and of the calculated sheath thickness for different values of $\xi$, in the $400 \mu \mathrm{m}$ diameter hole at $1 \mathrm{~mA}$.

We first see that the sheath size decays when $\xi$ increases. However, we note that for $\xi<0.5$, the influence on the calculated sheath thickness remains weak. We further observe that the maxima of both emission lines are located just after the sheath edge whatever the value of $\xi$ (with the exception of the case at 30 Torr, $\xi=0$ ). Moreover, the evolution of the sheath thickness with the pressure follows the same trends as that of the maxima of the emission lines.

The above model does not allow the possibility of a sheath free of ionization to be investigated. Indeed, letting, $\xi=1$ would result in a sheath thickness equal to zero. In the next section, we develop a model appropriate for this case.

\subsection{Case of a non ionizing sheath}

The calculation of the sheath size in a non-ionizing sheath can be performed in a self-consistent fashion. The model is known as the collisional Child-Law, and is described in text books [see for instance 18, page 185]. It consists of the following set of equations:

$$
\begin{cases}u_{i}=\mu_{i} E & (a) \\ J_{i}=e n_{i} u_{i} & (b) \\ \operatorname{div} J_{i}=0 & (c) \\ \operatorname{div} E=\frac{e n_{i}}{\varepsilon_{0}} & (d)\end{cases}
$$

The first equation (9a) indicates that the ion motion is limited by collisions with neutrals. The ion velocity $u_{i}$ is therefore a function of the electric field $E$ and of the ion mobility $\mu_{i}$. In the sheath, the reduced electric field is relatively high $\left(\mathrm{E} / \mathrm{p}>10^{3} \mathrm{~V} \cdot \mathrm{cm}^{-1}\right.$.Torr $\left.{ }^{-1}\right)$, such that the mobility is not a constant but varies with the ion fluid velocity [20, page 144]:

$$
\mu_{i}=\frac{2 e \lambda_{i}}{\pi m_{i} u_{i}},
$$

where $\mathrm{e}$ is the elementary charge, $\mathrm{m}_{\mathrm{i}}$ the ion mass and $\lambda_{\mathrm{i}}$ the ion-neutral mean free path. The second equation (9b) is simply the definition of the current density $J_{i}$, where $n_{i}$ the ion density. The third equation (9c) is a consequence of the assumption of non-ionizing sheath, and the last one (9d) is the Maxwell-Gauss law.

These four equations may be combined and integrated twice, in cylindrical coordinates, between R-d and $\mathrm{R}$ to obtain the electric field: 


$$
E(r)=\left[\sqrt{\frac{\pi m_{i}}{2 \lambda_{i} e}} \frac{I_{d} R}{S_{C}(1+\gamma) \varepsilon_{0}}\left(1-\left(\frac{R-d}{r}\right)^{3 / 2}\right)\right]^{2 / 3}
$$

To obtain this expression we have used the following boundary conditions,

$$
\left\{\begin{array}{l}
J_{i}(R)=\frac{I_{d}}{S_{C}(1+\gamma)} \\
E(R-d)=0
\end{array}\right.
$$

where $I_{d}$ is the discharge current and $S_{c}$ the surface on which the discharge current is collected at the cathode. Note that since the plasma expands on the cathode side, this surface is not equal to the cathode surface inside the hole. This is an important aspect of the following discussion.

Finally, the electric field is further integrated between R-d to R, to obtain the following integral equation:

$$
V_{d}=\int_{R-d}^{R}\left[\sqrt{\frac{\pi m_{i}}{2 \lambda_{i} e}} \frac{I_{d} R}{S_{C}(1+\gamma) \varepsilon_{0}}\left(1-\left(\frac{R-d}{r}\right)^{3 / 2}\right)\right]^{2 / 3} d r
$$

Here we have assumed that the potential drop across the sheath is equal to the discharge voltage $V_{\mathrm{d}}$. This equation is solved to obtain the evolution of the sheath thickness with the neutral gas pressure. The discharge voltage $V_{d}$ and the discharge current $I_{d}$ were maintained at $250 \mathrm{~V}$ and $1 \mathrm{~mA}$ respectively. The ion-neutral mean free path for charge exchange is taken to be $\lambda_{\mathrm{i}}=0.3 \mu \mathrm{m}$ at 100 Torr [20, page 80]. We note that $\mathrm{Ar}_{2}{ }^{+}$ions may also represent a significant fraction of the ion current and that consequently $\lambda_{\mathrm{i}}$ may be somewhat slightly different from our estimation.

An other issue is the determination of $\mathrm{S}_{\mathrm{C}}$, which is not independent of pressure due to the cathodic plasma expansion. To estimate the evolution of this parameter with pressure, we have measured the size of the region emitting light, noted $\mathrm{xR}$, on the cathode backside. Remembering that the cathode thickness is $\mathrm{L}=150 \mu \mathrm{m}$, the surface $\mathrm{S}_{\mathrm{C}}$ then reads

$$
S_{c}=2 \pi R L+\pi R^{2}\left(x^{2}-1\right)
$$

This provides an upper value for $S_{C}$ since $J_{i}$ is not uniform but may decay from the centre of the hole towards the edge of the emitting region.

Figure 7 shows the result of the calculation for $x=1$ (not including the backside expansion) and $x$ determined experimentally for each pressure $(x>1)$. The sheath size calculated with $x=1$ is near the maxima of the emission lines. However, we note that the trend with pressure does not agree well with experimental results. When $\mathrm{x}$ is a function of pressure, the sheath size is considerably larger, but the trend with pressure is in better agreement with our observations. However, these results are in contradiction with the model hypothesis. Indeed, the maximum of ionization must be near the maximum of the emission intensity of Ar line, which implies that ionization should take place within the sheath. This model seems inappropriate and consequently a large fraction of ionization is likely to occur within the sheath (as described in the previous model of section 4.1). 


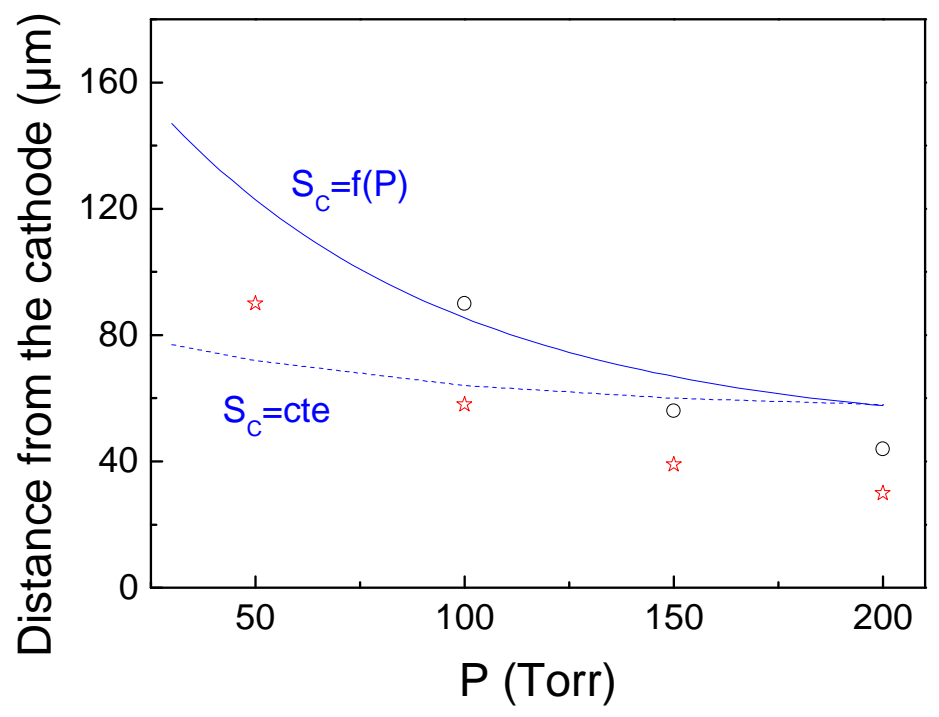

FIGURE 7. Position of the maxima (evaluated from the cathode) due to electron-impact excitation of the atomic line (black open circles) and of the ionic line (red open stars), as a function of pressure. The lines give the sheath thickness calculated for a non-ionizing sheath for $\mathrm{x}=1$ (blue dotted line) and for $\mathrm{x}=\mathrm{f}(\mathrm{P})$ (blue line). The hole diameter is $400 \mu \mathrm{m}$ and the discharge current is $1 \mathrm{~mA}$.

\section{Conclusions}

In this paper, we have used spectrally resolved imaging of the emission of the MHCD to study the spatial dependence of the excitation mechanism inside the discharge. The Micro Hollow Cathode Discharge was operated in the normal glow regime where the plasma does not stay confined inside the hole but also expands on the cathode backside. In addition, we proposed two sheath models to calculate the position of the sheath edge. The first model considers a fully ionizing sheath with a nonself consistent electric field, and the second considers a non-ionizing sheath with variable mobility and self-consistent calculation of the electric field. Our two main conclusions are:

(i) Both ionic and atomic lines present sharp electron impact excitation peaks near the cathode and the position of these peaks move towards the cathode as the pressure increases, in very good agreement with the position of the sheath edge calculated from the ionizing sheath model. The sheath edge calculated with the non-ionizing sheath model does not coincide with the position of the emission peaks, which suggests that a significant fraction of the ionization occurs in the sheath and consequently the ion flux produced in the sheath represents a significant fraction of the total ion flux at the cathode surface.

(ii) In addition to the sharp peaks near the plasma edge, the Ar emission line exhibits a dome-like profile, with a maximum in the discharge centre. This was attributed to the recombination of electrons with the molecular $\left(\mathrm{Ar}_{2}^{+}\right)$ions, a process that dominates in the discharge centre were the densities are maximal.

\section{References}

[1] Schoenbach K H, Verhappen R, Tessnow T, Peterkin F E and Byszewski W W 1996 Appl. Phys. Lett. 6813

[2] Adler F, Davliatchine and Kindel E 2002 J. Phys. D: Appl. Phys. 352291 
[3]Kushner M 2005 J. Phys. D: Appl. Phys. 381633

[4]Boeuf J.P, Pitchford L.C and Schoenbach K.H 2005 Appl. Phys. Lett. 86071501

[5] Kim G J, Iza F and Lee J K 2006 J. Phys. D: Appl. Phys. 394386

[6] Deconinck T. and Raja L.L. 2009 Plasma process. Polym. 6335

[7] Stoffels E, Flikweert A J, Stoffels W W, Kroesen G M W 2002 Plasma Sources Sci. Technol. 16383

[8] Rahul R, Stan O, Rahman A, Littlefield E, Hoshimiya K, Yalin A P, Sharla A, Pruden A, Moore C A, Yu Z and Collins G J 2005 J. Phys. D: Appl. Phys. 381750

[9] Moselhy M, Shi W and Stark Robert H 2002 IEEE Trans.Plasma Sci. 30198

[10] Mohan Sankaran R and Giapis Konstantinos P 2002 J.Appl. Phys. 922406

[11] Rousseau A and Aubert X 2006 J. Phys. D: Appl. Phys. 391619

[12] Aubert X, Bauville G, Guillon J, Lacour B, Puech V and Rousseau A 2007 Plasma Sources Sci. Technol. 1623

[13] A.I. Strinic, G.N. Malovic, Z.Lj. Petrovic and N. Sadeghi 2004 PSST 13333

[14]Johnsen R, Chen A and Biondi M.A 1980 J. Chem. Phys. 731717

[15]Hinnov E and Hirschberg J.G 1962 Phys. Rev. 125795

[16]Mehr F.J and Biondi M.A 1968 Phys. Rev. 176322

[17] K. Makasheva, J.-P. Boeuf and L.C. Pitchford, Topic 10, $28^{\text {th }}$ ICPIG, July 15-20, 2007, Prague, Czech Republic

[18]Roux F and Michaud F 1983 J. Mol. Spectroscopy 97253

[19]Piper L.G, Holtzclal K.W, Green B.D and Blumberg W.A.M 1989 J. Chem. Phys. 905337

[20] Lieberman M A and Lichtenberg A J 2005 Principle of Plasma Discharges and Materials Processing

[21]Cobine J.D. 1958 Gaseous Conductors Dover (New York) 220

[22] Debontride H, Derouard J, Edel P, Romestain R, Sadeghi N and Boeuf JP 1989 Physical Review A 405209 